\title{
¿Qué determina la realización de la prueba de VIH en la población panameña sexualmente activa entre los 15-59 años de edad? Hallazgos del análisis secundario de ENASSER 2009.
}

\author{
Lorna Jenkins $^{1, *}$, Ilenia Forero ${ }^{2}$, Elizabeth Castaño ${ }^{3}$, John \\ Hembling y Dora Estripeaut ${ }^{4}$ \\ ${ }^{1}$ Oficina Regional de Latinoamérica y el Caribe, Fondo de Población de las Naciones Unidas. \\ ${ }^{2}$ Oficina de planificación, Hospital del Niño, Panamá, República de Panamá. \\ ${ }^{3}$ Infectología, Hospital del Niño, Panamá, República de Panamá. \\ ${ }^{4}$ Jefa de Clínica de Terapia Antirretroviral, Hospital del Niño, Panamá, República de Panamá. \\ *Autor para correspondencia. Email: ljenkins@unfpa.org
}

Recibido: 15 de noviembre de 2014

Aceptado: 10 de diciembre de 2014

\begin{abstract}
Only $36.3 \%$ of HIV/AIDS diagnoses in Panama are made at an early stage. Thus, it is relevant to describe the factors that are associated with conducting HIV testing in sexually active individuals in Panama to design strategies for early detection of people living with HIV. This is a secondary analysis from the Sexual and Reproductive Health National Survey conducted in 2009. There were 7,359 cases identified to conduct further analysis, which were performed using the statistical analysis software Stata SE 13.0. Only $13.8 \%$ of men and $18.4 \%$ of women were tested for HIV in the twelve months preceding the survey. One in ten did it so voluntarily. Factors such as belonging to an indigenous group, or residing in an indigenous area are statistically associated to not performing the HIV test in men and women. Factors such as the number of sexual partners in the last 12 months, condom use and comprehensive knowledge of HIV are not statistically significant in either men or women. It is urgent to design strategies in order to increase the flow of people attending counseling services and HIV screening, especially in indigenous areas and in population self-identified as indigenous, as well as to increase knowledge and sexual risk behaviors associated to HIV.
\end{abstract}

Keywords: HIV testing; Voluntary test; HIV; AIDS; Comprehensive HIV knowledge; Panama. 
Invest. pens. crit.

Vol. 3, No. 1, enero-abril 2015

pp. 04-16

\section{Resumen}

En Panamá sólo el 36.3\% de los diagnósticos se hacen en la fase de VIH. Es necesario conocer los factores que se asocian a la realización de la prueba de VIH en personas con vida sexual activa en Panamá para diseñar estrategias para la detección temprana de las personas que viven con VIH. El presente estudio es un análisis secundario a partir de la Encuesta Nacional de Salud Sexual y Reproductiva 2009. Se analizaron las respuestas de 7,359 casos que reportaron tener vida sexual activa. Los análisis se realizaron con el programa de análisis estadístico Stata SE 13.0. El 13.8\% de los hombres y $18.4 \%$ de las mujeres se realizaron la prueba de VIH en los doce meses previos a la encuesta. Uno de cada diez lo hizo de manera voluntaria. Factores como pertenecer a un grupo indígena, o residir en un área comarcal se asoció de manera estadísticamente significativa a la no realización de la prueba tanto en hombres como en mujeres. Factores como el número de parejas sexuales en los últimos 12 meses, uso de condón y conocimientos comprehensivos de VIH no mostraron asociación estadísticamente significativa ni en hombres ni en mujeres. Consideramos urgente diseñar estrategias para aumentar el flujo de las personas que asisten a los servicios de asesoría y tamizaje de VIH, especialmente en las áreas indígenas y a la población auto-identificada como indígena.

Palabras clave: Prueba de VIH; Prueba voluntaria; VIH; SIDA; Conocimiento comprehensivo de VIH; Panamá

\section{Introducción.}

Panamá, con una prevalencia de $0.9 \%$, es el segundo país más afectado con VIH en la región de Centroamérica (UNAIDS, 2013). La epidemia de VIH/SIDA en Panamá es de tipo concentrada ya que la población mayormente afectada son los HSH con 12.4\% (mayor al 5\%) y entre las embarazadas la afectación es menor al 1\% (0.3\%). Panamá ha sufrido una rejuvenizacion y feminización de la epidemia del VIH desde 1994, momento en que la tasa de mujeres viviendo con VIH era de 2.4/100 000 y actualmente se encuentra en 30.2/100 000. La relación hombre mujer es 3.0:1.0 en población general, mientras que en el grupo de 15-24 años hay dos veces más mujeres afectadas que hombres (Ministerio de Salud [MINSA] de Salud, Departamento de Epidemiología, 2013).

Hasta diciembre del 2012, en Panamá se han diagnosticado 20569 casos de VIH/sida. El 36.3\% de los casos fueron detectados en fase VIH y el 63.7\% en fase sida (MINSA, Departamento de Epidemiología, 2013). Debido a la importancia que existe en el diagnóstico temprano e inicio de tratamiento antirretroviral (TARV) para mejorar el pronóstico de las personas que viven con VIH consideramos importante analizar los factores que se asocian a la realización de la prueba de VIH.

La calidad y cobertura de los servicios de asesoría y prueba de VIH deben ser fortalecidas para la tamización voluntaria de los ciudadanos (MINSA, Programa Nacional de ITS/VIH/SIDA, 2013). Al conocer su estado inmunológico de manera oportuna, los mismos puedan ser referidos a las clínicas de terapia antirretroviral (CTARV) para el inicio de tratamiento. Los factores sociales, económicos y conductuales que se asocian a la realización de la prueba deben ser estudiados con miras a formular 
Invest. pens. crit.

Vol. 3, No. 1, enero-abril 2015

pp. 04-16

políticas basadas en evidencia para mejorar la sobrevida y calidad de vida de aquellos que viven con VIH.

La literatura describe que la realización de la prueba de VIH se asocia con:

- El número de parejas sexuales. Un estudio realizado entre estudiantes universitarios de Perú mostro que un $20 \%$ de los participantes en el estudio se consideraban con riesgo de contagio por VIH alto o moderado por contar con más de un compañero sexual (Alcarraz, Arias, Peña, Ventura \& Calle, 2008).

- La no utilización del condón de manera consistente. El mismo estudio menciona que el no uso de condón es percibido con un riesgo de contagio por VIH alto o moderado (Alcarraz et al, 2008).

- Etnia. En el estudio de Taylor, Hembling y Bertrand (2014) realizado en Guatemala, los hombres y mujeres auto-identificados/as como pertenecientes a una etnia indígena reportaron nunca haberse realizado la prueba de VIH en más del 94.0\% de los casos en comparación con el grupo de ladinos que fue entre $78.7 \%$ en mujeres y $82.1 \%$ en hombres de las mismas áreas estudiadas.

El objetivo principal de este análisis secundario es conocer los factores que influyen en la realización de la prueba de VIH en los doce meses previos a la encuesta ENASSER 2009 entre los hombres y mujeres sexualmente activos que participaron.

\section{Materiales y métodos}

\section{Muestra}

La ENASSER de 2009 es la primera encuesta nacional dedicada al tema de salud sexual y reproductiva, que incluyó una sección sobre conocimientos del VIH/sida. El marco de muestreo para esta encuesta estuvo basado en el X Censo de Población y VI de Vivienda de la República de Panamá del año 2000.

Para esta encuesta la unidad primaria de muestreo es el segmento censal; la unidad secundaria de muestreo es la vivienda y la unidad de observación corresponde a las mujeres en el rango de 15 a 49 años de edad, que se encuentren en los dos primeros segmentos seleccionados y los hombres de 15 a 59 años de edad que se encuentren en cada tercer segmento seleccionado.

\section{Definición de variables}

La variable dependiente del estudio es la realización de la prueba de VIH en los últimos 12 meses: Se considera una respuesta afirmativa si el sujeto respondió en la encuesta ENASSER 2009 que se la realizó en los doce meses previos al momento de la entrevista.

Los variables independientes que estudiamos son: 
- Etnia. Es una comunidad humana definida por afinidades raciales, lingüísticas, culturales (Real Academia de la Lengua Española [RAE], 2012). Para efectos de este análisis los individuos serán clasificados como no indígenas, que incluye a todos aquellos que no se consideren indígenas o de algún otro grupo étnico, o indígenas, que incluye los 9 grupos existentes en Panamá (Instituto Nacional de Estadísticas y Censo [INEC], 2010).

- Área de residencia. Se refiere a una localidad separada físicamente de otra (INEC, 2010) y que según las características de las viviendas y el acceso a bienes. Se clasificó en urbano, rural e indígena.

- Nivel de escolaridad. Se refiere al grado o año escolar más alto aprobado dentro de los niveles que se imparten en escuelas y universidades públicas y privadas del país (INEC, 2010). Para efectos del análisis se agruparon en: sin escolaridad o primaria, hasta secundaria y con educación superior.

- Religión. Es el conjunto de creencias o dogmas acerca de la divinidad, de normas morales para la conducta individual y social y de prácticas rituales (RAE, 2012). Según las religiones establecidas en el país tomadas en cuenta para esta encuesta se agruparon en: católica, cristiana, otra o ninguna.

- Inicio de vida sexual. Para fines de este análisis se definió como antes o a los 15 años de edad, entre los 16 y 17 años de edad, o a los 18 o más años de edad.

- Número de parejas sexuales en los últimos 12 meses. Para fines de este análisis se definió agrupando las respuestas según 1 o menos parejas, o respondiendo 2 o más parejas. Esta agrupación se definió tomando en consideración todas las respuestas posibles dadas por los encuestados y buscando la media de la misma.

- Conocimiento comprehensivo de VIH. Es saber distinguir correctamente las formas de prevenir la transmisión sexual del virus de VIH y rechazar conceptos erróneos sobre dicha transmisión. Para fines de este análisis se tomaron en cuenta las respuestas correctas a 3 preguntas sobre prevención y 2 preguntas sobre rechazo de ideas erróneas.

\section{$\underline{\text { Análisis estadístico }}$}

El análisis estadístico se realizó con el programa informático Stata SE 13.0. Los datos fueron obtenidos de la base de datos resultante de la encuesta ENASSER 2009.

A través de la revisión de los resultados de la encuesta y de identificación de variables necesarias se procedió a ajustarlas según las variables dependientes e independientes que deseamos estudiar. Una vez creadas y categorizadas las variables, se realizaron las asociaciones correspondientes para determinar si existían diferencias. Estas diferencias fueron analizadas con Chi cuadrado de Pearson o el Test de Wald dependiendo de la cantidad de categorías que contenía la variable para encontrar en cuales categorías existía la diferencia estadística. 
Invest. pens. crit.

Vol. 3, No. 1, enero-abril 2015

pp. 04-16

\section{$\underline{\text { Limitaciones }}$}

Por ser este un análisis secundario presentamos las siguientes limitaciones:

- Análisis de una encuesta transversal. Sólo se pudieron examinar asociaciones entre las variables previamente definidas seleccionadas en base a nuestro interés, es decir, no permitió realizar un análisis de causalidad.

- La metodología de recolección de los datos. La encuesta se realizó cara a cara y los sujetos debían auto-reportar conductas sexuales, por lo que puede existir sesgo sistemático en las respuestas.

- El análisis bivariado también constituye una limitante en el presente análisis. Este tipo de análisis permite realizar un tratamiento estadístico a dos variables, pero no podemos estimar si otra variable además de estas primeras dos, influye en tal asociación.

\section{Resultados}

Características de la población estudiada.

Para este análisis se seleccionaron hombres entre 15 y 59 años de edad y mujeres entre 15 y 49 años de edad que reportaron haber iniciado vida sexual.

De las 8,662 personas encuestadas, 85\% (7359) reportaron haber iniciado vida sexual (2,456 hombres y 4,903 mujeres). El 68\% de estos hombres y un 69.4\% de estas mujeres vivían en áreas urbanas, mientras que el $32 \%$ de estos hombres y $30.6 \%$ de estas mujeres se concentraban en las áreas rural y comarcas. El 89.5\% de los hombres y el 90.1\% de las mujeres no pertenecían a ningún grupo indígena.

Es notable que el $52.1 \%$ y $48.9 \%$ de hombres y mujeres completaron hasta secundaria, así como un $19.8 \%$ y $24.8 \%$ de hombres y mujeres llegaron a una educación superior. El mayor grupo religioso reportado fue el católico con un $74.5 \%$ en los hombre y $70.4 \%$ en las mujeres.

En cuanto a la sexualidad, un $75.6 \%$ de los hombres y $51.4 \%$ de las mujeres reportaron haber iniciado vida sexual antes de los 18 años de edad. Tanto en el grupo de hombres como en el de mujeres predominó el reporte de 1 o menos parejas sexuales en los últimos 12 meses con 75.5\% y 92.2\% respectivamente.

Para la evaluación de prácticas relacionadas con sexo, al preguntar por la realización de la prueba de VIH en los últimos 12 meses sólo 13.8\% de los hombres y 18.4\% de las mujeres se la habían realizado. Entre las mujeres, sólo un 10.2\% se la realizó de forma voluntaria. Al analizar las mujeres sexualmente activas según la realización de la prueba de VIH en los 12 meses previos a la encuesta encontramos que el $89.8 \%$ de las mismas no se realizaron la prueba de VIH de forma voluntaria. Los hombres sexualmente activos refirieron en un $86.2 \%$ no haberse realizado la prueba en los 12 meses previos a la aplicación de la encuesta. 
Los conocimientos comprehensivos de VIH fueron muy parecidos para ambos sexos, se encontró una deficiencia de conocimiento de $83.3 \%$ en los hombres y $80.8 \%$ en las mujeres.

En relación al uso de anticonceptivos modernos el 71.1\% de los hombres reportaron haber usado alguna vez el condón, y de estos solo 15.2\% lo utilizan con frecuencia. Mientras que el 53.8\% de las mujeres refiere estar utilizando algún método anticonceptivo en la actualidad.

Realización de la prueba de VIH en los últimos 12 meses en los hombres y mujeres sexualmente activos/as

Si evaluamos las mujeres que se realizaron la prueba de VIH en los doce meses previos a la encuesta según la etnia, el 90.8\% de las indígenas y el $80.6 \%$ de las mujeres no indígenas no se realizaron la prueba en los últimos 12 meses (p<.05). Entre los hombres, el 85.8\% de los no indígenas y el 89.1\% de los indígenas no se realizó la prueba, diferencia que no es estadísticamente significativa.

Figura 1. Realización de la prueba de VIH en mujeres de 15 a 49 años de edad según área de residencia

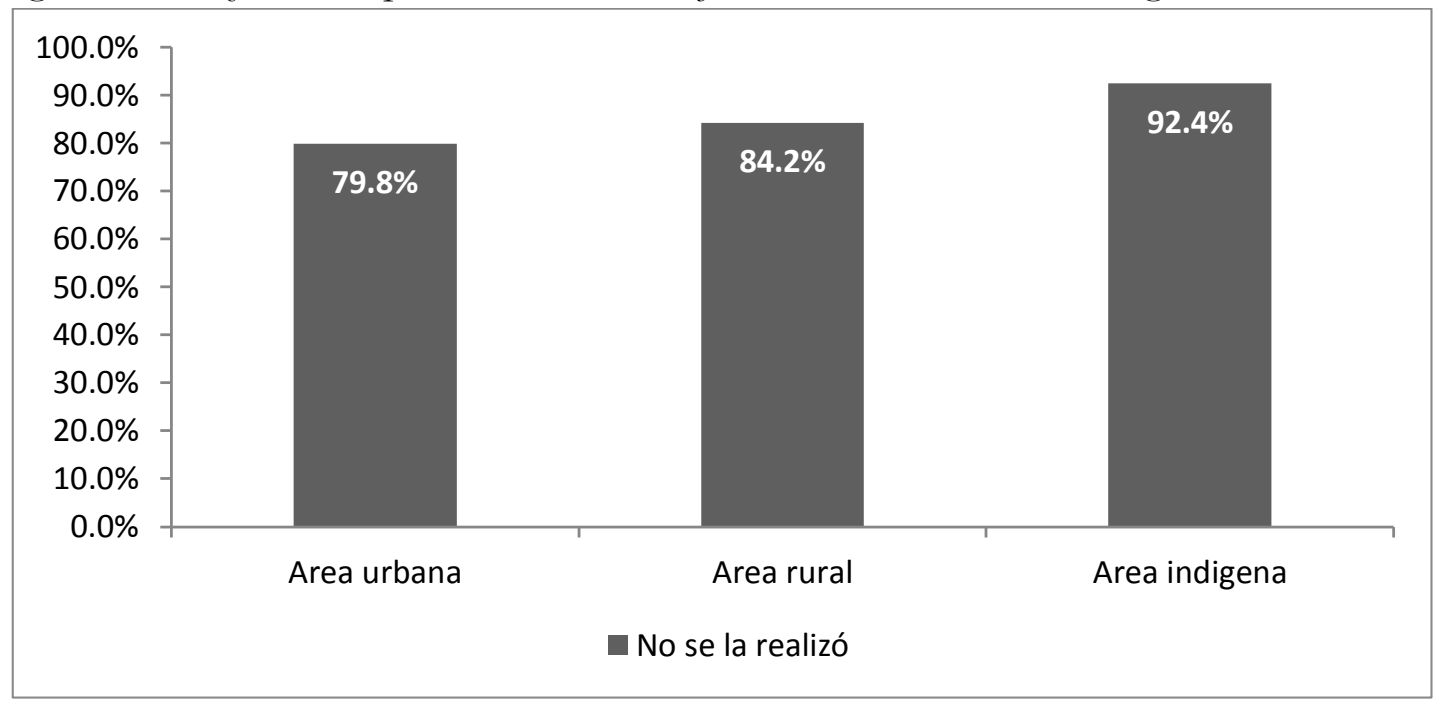

Nota: $\mathrm{p}<.05$.

De igual forma no se realizaron la prueba de VIH las mujeres que residen en área comarcal en un $92.4 \%$, las que residen en un área rural en un $84.2 \%$ y las que viven en el área urbana 79.8\%. La diferencia entre aquellas que viven en el área urbana versus el comarcal (Figura 1) fue significativa $(\mathrm{p}<.05)$. Entre los hombres la mayor tendencia a no realizarse la prueba se encontró en el grupo que reside en el área indígena con 93.4\%, sin embargo, los que residen en área urbana y rural también contaban con un alto porcentaje, $84 \%$ y $90.3 \%$ respectivamente (Figura 2), hallazgo que es estadísticamente significativo $(\mathrm{p}<.05)$. 
Invest. pens. crit.

Vol. 3, No. 1, enero-abril 2015

pp. 04-16

Figura 2. Realización de la prueba de VIH en hombres de 15 a 59 años de edad según área de residencia

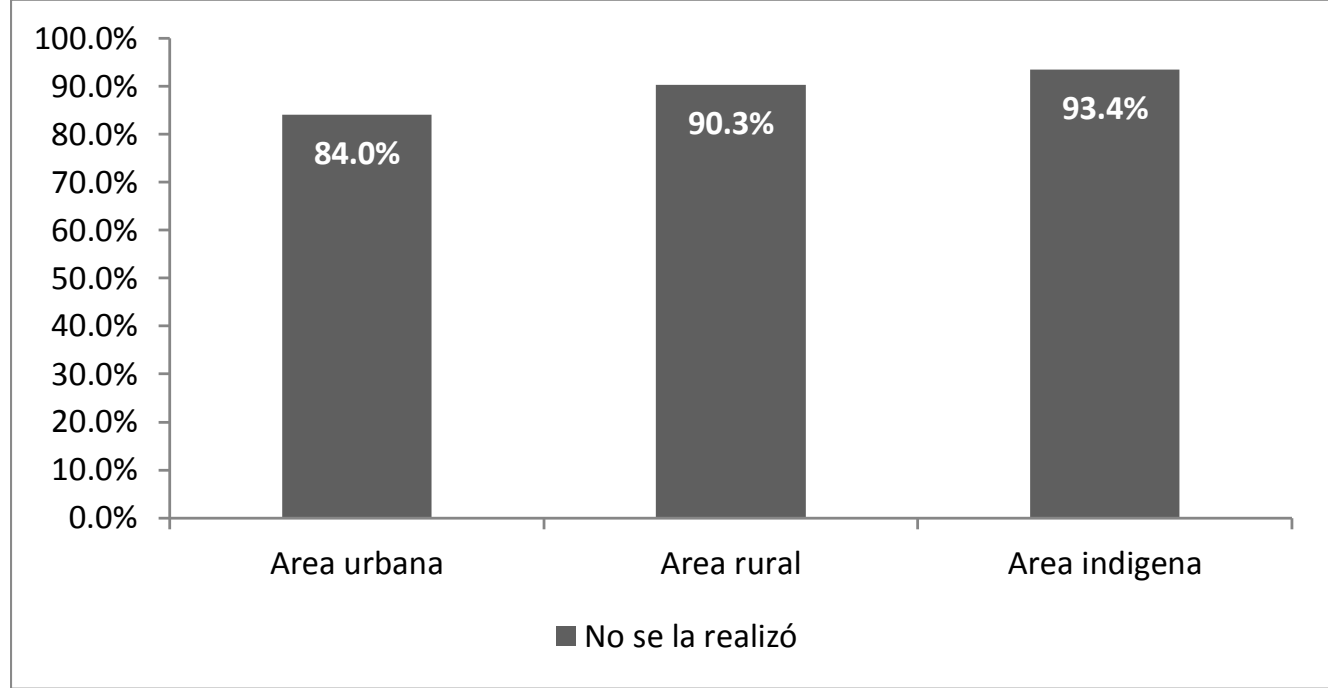

Nota: $\mathrm{p}<.05$.

Indistintamente del sexo, escolaridad y de la religión, no se realizaron la prueba en $80 \%$ o más de los que respondieron la encuesta ENASSER 2009. Sin embargo, tanto hombres como mujeres con educación primaria o menos no se realizaron la prueba de VIH en los doce meses previos a la encuesta ENASSER 2009, lo que resultó estadísticamente significativo $(\mathrm{p}<.05)$. Entre las mujeres que no reportaron practicar religión también se halló una diferencia estadísticamente significativa para la no realización de la prueba de VIH ( $\mathrm{p}<.05)$.

Figura 3. Realización de la prueba de VIH en mujeres de 15 a 49 años de edad según edad de inicio de vida sexual

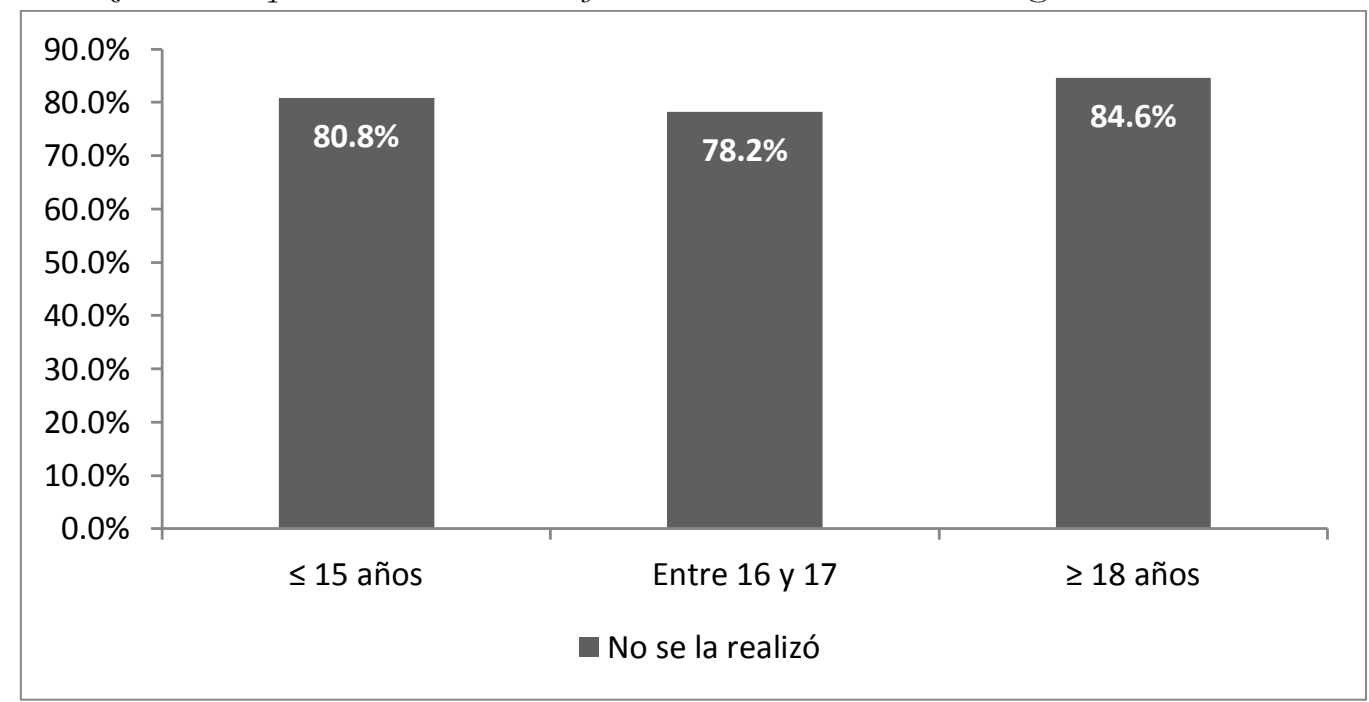

Nota: $\mathrm{p}<.05$.

$\mathrm{Al}$ analizar la realización de la prueba de VIH y edad de inicio de vida sexual (Figura 3) se encontró una diferencia estadísticamente significativa $(\mathrm{p}<.05)$ entre aquellos que iniciaron vida sexual antes de 
los 15 años y la no realización de la prueba del VIH (81.9\% de hombres y 80.8\% de las mujeres). Es importante resaltar que indistintamente de la edad de inicio de vida sexual más del $80 \%$ refirieron no realizarse la prueba de VIH (Gráfica 4).

Figura 4. Realización de la prueba de VIH en hombres de 15 a 59 años de edad según edad de inicio de vida sexual

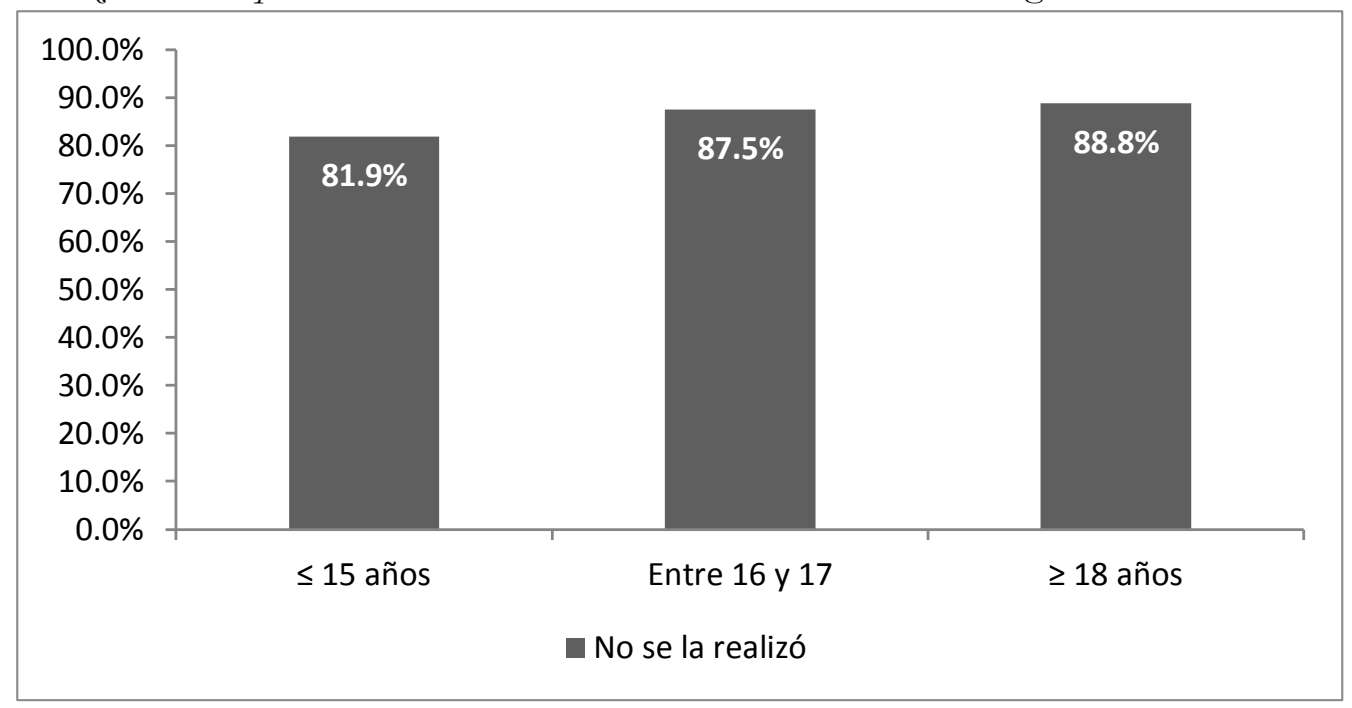

Nota: $\mathrm{p}<.05$.

En mujeres el número de parejas sexuales en los últimos 12 meses, uso actual de métodos anticonceptivos modernos y conocimientos comprehensivos de VIH no se asociaron a la realización de la prueba del VIH en los doce meses previos a la encuesta ENASSER 2009. Sin embargo podemos decir que en todos estos factores un alto porcentaje de las mujeres manifestó no haberse realizado la prueba en los últimos 12 meses (Tabla 1).

$\mathrm{Al}$ analizar en los hombres la realización de la prueba de VIH los 12 meses previos a la encuesta con el número de parejas sexuales y los conocimientos comprehensivos de VIH no se encontró asociación estadística, pero la tendencia es no realizarse la prueba (Tabla 1). Al explorar el uso del condón, se encontró que el $90.7 \%$ nunca ha usado el condón y no se ha realizado la prueba de VIH en los doce meses previos, hallazgo que fue estadísticamente significativo. Sin embargo la frecuencia de uso del condón no se asocia estadísticamente a la realización de la prueba del VIH en los doce meses previos a ser entrevistados.

\section{Discusión: Implicaciones programáticas}

\section{Educación sexual desde temprana edad para ambos sexos.}

El análisis encontró que el inicio de la actividad sexual en Panamá es antes de los 18 años (75.6\% en los hombres y $51.4 \%$ en las mujeres), igualmente se evidenciaron bajos conocimientos comprehensivos de VIH y la identificación de conductas sexuales de riesgo. 
Invest. pens. crit.

Vol. 3, No. 1, enero-abril 2015

pp. 04-16

Tabla 1. Realización de la prueba de VIH en los últimos 12 meses en hombres y mujeres sexualmente activos.

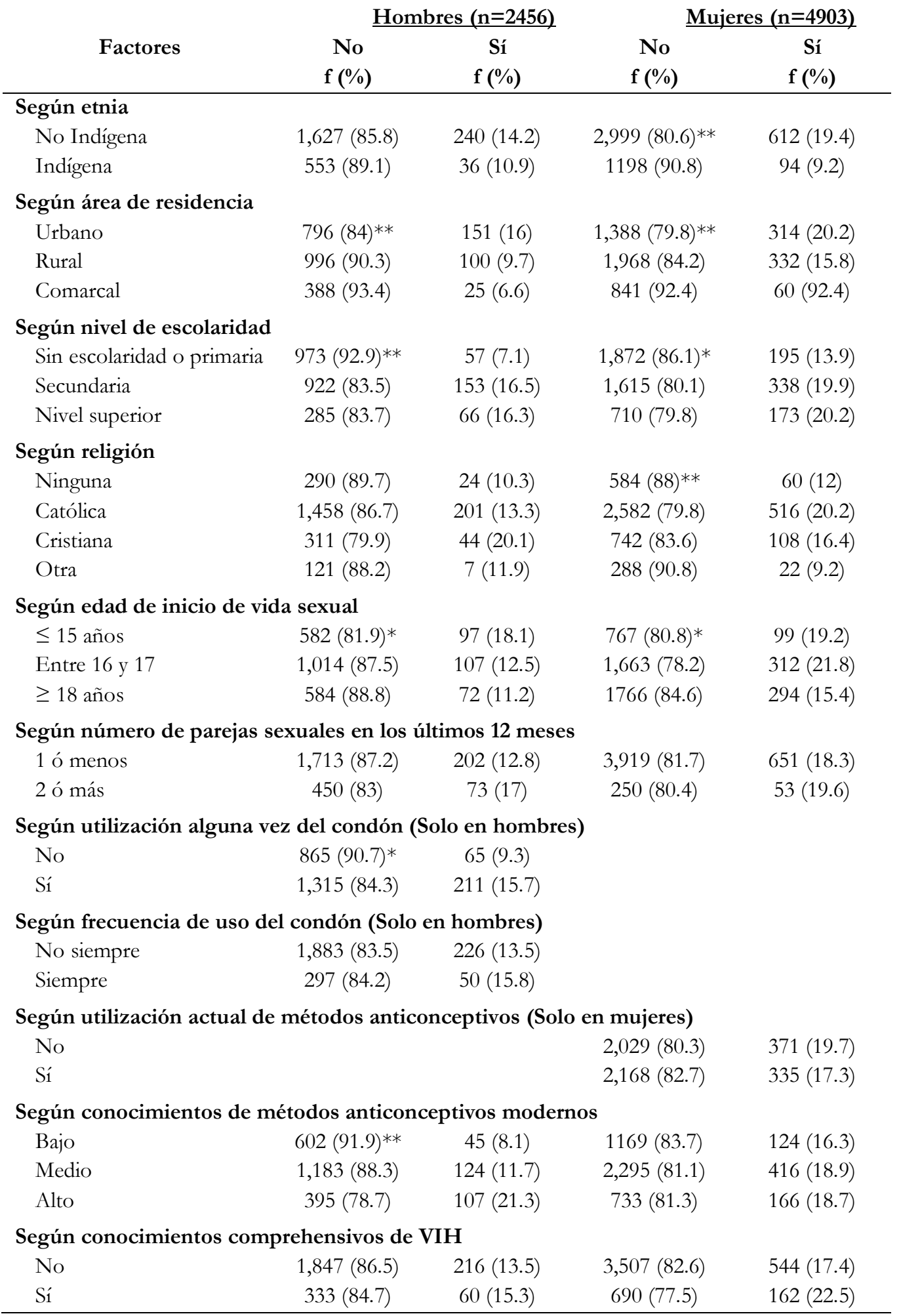

Nota: ${ }^{*} \mathrm{p}<.05 ; * * \mathrm{p}<.01$ 
Invest. pens. crit.

Vol. 3, No. 1, enero-abril 2015

pp. $04-16$

Es necesario desarrollar currículos que promuevan el autocuidado de la salud, incluida la práctica responsable de la sexualidad con enfoque de género y derechos humanos; que habiliten a las personas a retardar el inicio de la actividad sexual, como también disminuir el número de parejas sexuales en un año.

Promover la revisión del contenido y actualización de la metodología para brindar información a los usuarios de los programas de atención de salud sexual y reproductiva e ITS/VIH/SIDA

El análisis demostró tanto en las mujeres como en los hombres que no hay asociación entre tener 2 o más parejas sexuales y el conocimiento comprehensivo de VIH con la realización de la prueba de VIH en los 12 meses previos a la encuesta. El 83.0\% de los hombres y el 80.4\% de las mujeres que tuvieron dos o más parejas sexuales no se realizaron la prueba. La realización de la prueba de VIH en los 12 meses previos a la encuesta si se asocia al uso de condón en los hombres. Esto nos revela la oportunidad de implementar programas cognitivo-conductuales, en donde se brinde conocimiento sobre la transmisión de VIH y el correcto uso de anticonceptivos modernos. Deben reforzarse las conductas que ayuden a preservar la salud de los individuos como disminuir el número de parejas sexuales y la realización de la prueba de VIH de manera voluntaria.

Cambiar el discurso para promover la tamización voluntaria de la población.

En general, los hombres y mujeres sexualmente activos se realizaron la prueba de forma voluntaria solamente entre el $13.8 \%$ y $18.4 \%$, respectivamente. Es necesario establecer campañas de forma permanente en los diferentes medios, impresa, televisiva, radial sobre la importancia del conocimiento acerca de VIH y la realización de la prueba de VIH, desde la perspectiva del auto-cuidado de la salud, más que resaltando conductas de riesgo que promueven la estigmatización y miedo del individuo. Se debe continuar y ampliar las campañas de tamizaje gratuitas ya establecidas para embarazadas, población clave, y poblaciones que viven en situación de vulnerabilidad como lo son los hombres y mujeres que vivan en áreas indígenas o se auto-identifiquen en este grupo.

\section{Agradecimientos}

El estudio fue realizado gracias al apoyo del Programa Nacional de ITS/VIH/SIDA del Ministerio de Salud y la Sección de Salud Sexual y Reproductiva del Instituto Conmemorativo Gorgas de Estudios en Salud, especialmente al Dr. Aurelio E. Núñez M. y a la Dra. Ruth De León, respectivamente. Deseamos destacar el esfuerzo de Tulane University y USAID por fortalecernos técnicamente en el uso de Stata SE v 13.0.

\section{Declaración de los autores}

El resultado de la investigación y el contenido de la publicación son responsabilidad de los autores, no refleja la postura final de las instituciones para las cuales laboran. 
Invest. pens. crit.

Vol. 3, No. 1, enero-abril 2015

pp. $04-16$

\section{Referencias}

Alcarraz, C., Arias, K., Peña, C., Ventura, J., \& Calle, M. (2008). Factores Asociados a la realización de una prueba de detección de VIH en estudiantes universitarios. CIMEL, 13(2), 53-61.

Banco Mundial. (2013). Panamá. Disponible en http://data.worldbank.org/country/panama

Carrasco-Garrido, P., López de Andrés, A., Hernández Barrera, V., Jiménez-Trujillo, I., Santos-Sancho, J., \& Jiménez-García, R. (2011). Predictors of contraceptive methods among adolescents and young women residing in Spain. J Sex Med, 8, 2431-2438. Disponible en: http://dx.doi.org/10.1111/j.1743-6109.2011.02346.x

De la Cruz-Peñarán, D., Langer-Glas, A., Hernández-Prado, B., \& González-Rengijo, G.F. (2003). Conocimientos y actitudes de la pareja hacia la práctica de la planificación familiar en la Selva del Perú. Salud Publica Mex, 45, 461-471. Disponible en http://bvs.insp.mx/rsp/inicio/

Etnia. (2012). En Diccionario de la Real Academia de la Lengua Española en línea. Disponible en http://lema.rae.es/drae/?val=etnia

Fétis, G., Bustos, L., Lanas, F., Baeza, B., Contreras, J., Hebel, E., \& Marucich, C. (2010). Factores asociados al uso de anticonceptivos en estudiantes de enseñanza media de la comuna de Temuco. Rev Chilena de Obstetricia y Ginecología, 73(6), 362-369.

González, E., Caba, F., Molina, T., Sandoval, J., \& Meneses, R. (2003). Factores personales asociados al uso de anticonceptivos en adolescentes mujeres solteras sexualmente activas. Revista de la Sociedad Chilena de Obstetricia y Ginecología Infantil y de la Adolescencia, 10(2). Disponible en http://www.cemera.cl/sogia/pdf/2003/X2factores.pdf

González, M. (2011). Patrones de uso de anticonceptivos en diversos tipos de clínicas conforme la edad y género de los participantes, Programa de Planificación Familiar Título X del Recinto de Ciencias Médicas de la Universidad de Puerto Rico: 2007-2008. CIDE digital, 2(1), 53-74. Disponible en http://demografia.rcm.upr.edu/index.php/cide-digital

Instituto Nacional de Estadísticas y Censo. (2010). Definiciones y explicaciones utilizadas para el Censo de 2010. Panamá: Contraloría General de la República de Panamá. Disponible en http://www.contraloria.gob.pa/inec/archivos/P3561Definiciones.pdf

Joint United Nations Programme on HIV/AIDS (UNAIDS). (2013). Global report: UNAIDS report on the global AIDS epidemic 2013. Disponible en

http://www.unaids.org/sites/default/files/media asset/UNAIDS Global Report 2013 e n 1.pdf 
Invest. pens. crit.

Vol. 3, No. 1, enero-abril 2015

pp. $04-16$

León, F., Roca, S., \& Ríos, A. (2000). La consejería balanceada aumenta la calidad de la atención y el conocimiento de las mujeres sobre anticonceptivos. Population Council 2000. Disponible en http://www.popcouncil.org/pdfs/frontiers/FR.final

Ministerio de Salud, Departamento de Epidemiologia. (2013). Informe acumulativo de la situación de VIH/SIDA 1984-2012. Panama: Autor.

Ministerio de Salud, Programa Nacional de ITS/VIH/SIDA. (2013). Normas y directrices para la prueba y asesoría del VIH en Panamá. Panamá: Autor.

Organización Mundial de la Salud (OMS). (2014). Datos del Observatorio mundial de la salud [Repositorio de datos]. Disponible en http://apps.who.int/gho/data/?theme=main

Organización Panamericana de la Salud (OPS). (2013). Iniciativa Regional de Datos Básicos en Salud. [Sistema Generador de Tablas]. Disponible en http://www1.paho.org/English/SHA/coredata/tabulator/new'Tabulator.htm

Reis, M., Ramiro, L., Gaspar, M., \& Alves, J. (2013). Nationwide survey of contraceptive and sexually transmitted infection knowledge, attitudes and skills of university students in Portugal. Int J Clin Health Psychol, 13(2), 127-137.

Religión. (2012). En Diccionario de la Real Academia de la Lengua Española en línea. Disponible en http://lema.rae.es/drae/?val=religi $\%$ C $3 \%$ B 3 n

Schiappacasse, V., Vidal, P., Casas, L., Dides, C. \& Díaz, S. (2003). Chile: Situación de la saludy derechos sexuales y reproductivos. Santiago de Chile: Instituto de Medicina Reproductiva.

Schwab, K. (Ed). (2013). The Global Competitiveness Report 2013-2014. Geneva, Suiza: World Economic Forum. Disponible en http://www.weforum.org/reports/global-competitiveness-report-2013-2014

Taylor, T., Hembling, J., \& Bertrand, J. (2014). Ethnicity and HIV risk behavior, testing and knowledge in Guatemala. Ethnicity \& Health, 20(2), 263-177. Disponible en http://dx.doi.org/10.1080/13557858.2014.893562

Tilford, S., Clifton, J., Brice, L., Wiggins-Smith, J., Penrose, L., \& Farooqi, N. (2013). Influence of sex education on condom knowledge and use proficiency among Texas college students. Contraception, 88(3), 473.

Tilford, S., Clifton, J., Brice, L., Wiggins-Smith, J., Penrose, L., \& Farooqi, N. (2013). Influence of sex education on condom knowledge and use proficiency among Texas college students. Contraception, 88(3), 473. 
Invest. pens. crit.

Vol. 3, No. 1, enero-abril 2015

pp. $04-16$

Trejo-Ortiz, P., Moreno-Chávez, P., Macías-Aguilar, M., Valdez-Esparza, G., Mollinedo, F., Lugo, L., \& Araujo, R. (2011). Conocimiento y comportamiento sexual en adolescentes. Área Académica Ciencias de la Salud. Universidad Autónoma de Zacatecas. Revista Cubana de Enfermería, 27(4), 273-280.

Vázquez, A., \& Suárez, N. (2006). Conocimiento y uso de métodos anticonceptivos en la población de 15 a 44 años del área urbana, Cunduacán. Tabasco 2005. Horizonte Sanitario, 5(2), 8-25. 